\title{
Differential expression of tescalcin by modification of promoter methylation controls cell survival in gastric cancer cells
}

\author{
TAE WOO KIM ${ }^{1,2 *}$, SEUNG RO HAN ${ }^{3,4^{*}}$, JONG-TAE KIM ${ }^{1}$, SEUNG-MIN YOO ${ }^{3,4}$, MYUNG-SHIN LEE ${ }^{3,4}$, \\ SEUNG-HOON LEE ${ }^{3,5}$, YUN HEE KANG ${ }^{3,4}$ and HEE GU LEE ${ }^{1,2}$ \\ ${ }^{1}$ Immunotherapy Convergence Research Center, Korea Research Institute of Bioscience and Biotechnology; \\ ${ }^{2}$ Department of Biomolecular Science, University of Science and Technology (UST), Daejeon 34141; \\ ${ }^{3}$ Eulji Biomedical Science Research Institute; ${ }^{4}$ Department of Microbiology and Immunology; \\ ${ }^{5}$ Department of Neurosurgery, Eulji University School of Medicine, \\ Daejeon 34824, Republic of Korea
}

Received October 25, 2018; Accepted April 2, 2019

DOI: 10.3892/or.2019.7099

\begin{abstract}
The EF-hand calcium binding protein tescalcin (TESC) is highly expressed in various human and mouse cancer tissues and is therefore considered a potential oncogene. However, the underlying mechanism that governs TESC expression remains unclear. Emerging evidence suggests that TESC expression is under epigenetic regulation. In the present study, the relationship between the epigenetic modification and gene expression of TESC in gastric cancer was investigated. To evaluate the relationship between the methylation and expression of TESC in gastric cancer, the methylation status of $\mathrm{CpG}$ sites in the TESC promoter was analyzed using microarray with the Illumina Human Methylation27 BeadChip (Human Methylation27_270596_v.1.2), gene profiles from the NCBI Dataset that revealed demethylated status were acquired, and real-time methylation-specific PCR (MSP) in gastric cancer cells was conducted. In the present study, it was demonstrated that the hypermethylation of TESC led to the downregulation of TESC mRNA/protein expression. In addition, 5-aza-2c-deoxycytidine (5'-aza-dC) restored TESC expression in the tested
\end{abstract}

Correspondence to: Dr Yun Hee Kang, Eulji Biomedical Science Research Institute, Eulji University School of Medicine, Daejeon 34824, Republic of Korea

E-mail: yhkang@eulji.ac.kr

Dr Hee Gu Lee, Immunotherapy Convergence Research Center, Korea Research Institute of Bioscience and Biotechnology, Daejeon 34141, Republic of Korea

E-mail: hglee@kribb.re.kr

${ }^{*}$ Contributed equally

Abbreviations: TESC, tescalcin; MBD, methyl-CpG binding domain protein; HDAC, histone deacetylase; $\mathrm{CpG}$, cytosine guanine dinucleotide; MSP, methylation-specific PCR; RT-PCR, reverse transcription-polymerase chain reaction

Key words: gastric cancer, epigenetics, promoter methylation, MBD1, TESC gastric cancer cells except for SNU-620 cells. ChIP assay further revealed that the methylation of the TESC promoter was associated with methyl-CpG binding domain protein (MBD)1, histone deacetylase (HDAC)2, and Oct-1 and that treatment with 5'-aza-dC facilitated the dissociation of MBD1, HDAC2, and Oct-1 from the promoter of TESC. Moreover, silencing of TESC increased MBD1 expression and decreased the H3K4me2/3 level, thereby causing transcriptional repression and suppression of cell survival in NCI-N87 cells; conversely, overexpression of TESC downregulated MBD1 expression and upregulated the $\mathrm{H} 3 \mathrm{~K} 4 \mathrm{me} 2$ level associated with active transcription in SNU-638 cells. These results indicated that the differential expression of TESC via the modification status of the promoter and histone methylation controled cell survival in gastric cancer cells. Overall, the present study provided a novel therapeutic strategy for gastric cancer.

\section{Introduction}

It has been reported that tescalcin (TESC) is upregulated by treatment with class I histone deacetylase (HDAC) inhibitors. In addition, TESC plays important roles related to chromatin remodeling, transcriptional regulation, and epigenetic modification (1) by interacting with the cytosolic tail domain of $\mathrm{Na}^{+} / \mathrm{H}^{+}$exchanger isoform type-1 (NHE1), implicating novel gene functions in the regulation of NHE1 in cardiac tissues (2). This finding was further supported by a study that revealed that $\mathrm{Na}^{+} / \mathrm{H}^{+}$exchanger was regulated by both post-translational modifications and a number of regulatory binding proteins, including calmodulin, moesin and TESC (3). Notably, it was demonstrated that NHE1 was directly associated with cellular transformation, invasion and metastasis in cancer, suggesting that NHE1 is a potential novel drug target for anticancer therapeutics (4), and this was further supported by the observation of TESC overexpression in melanoma and colorectal and gastric cancers (5-7). However, the regulation of TESC expression in cancer and its underlying mechanisms are largely unknown.

TESC overexpression in K562 cells has been demonstrated to regulate cell proliferation via activation of ERK signaling, while the expression of Ets transcription factors, 
including Ets-1, Ets-2, and Fli-1, was blocked at the mRNA level following TESC knockdown (8). The Ets gene family belongs to one of the largest family of transcriptional targets in cancer (9-12). Recent research further indicated that high levels of DNA methylation were frequently observed in the binding sites of Ets transcription factors, including PDEF, GABPA, ELF1, FLI1 and ETS2 (13).

Epigenetic alterations caused by the methylation of cytosine guanine dinucleotide $(\mathrm{CpG})$ islands are reported to play pivotal regulatory roles in gene expression, differentiation, apoptosis and tumorigenesis (14,15). In addition, promoter methylation is considered an important regulatory mechanism for the expression of gastric cancer-related genes, such as NDRG2, MGMT, hMLH1, RASSF1A, p16 and RUNX3, which have been revealed to be involved in gastric carcinogenesis $(16,17)$. Furthermore, tumor suppressor genes and oncogenes are mainly detected in repetitive sequences within the genome as well as in regions of hypermethylation where the gene promoters are located with $\mathrm{CpG}$ islands. Notably, promoter hypomethylation has been revealed to be associated with oncogene activation, whereas hypermethylation of CpG sites was revealed to be associated with inactivation of tumor-suppressor genes (18-21). In addition, DNA methylation may inhibit gene expression by either blocking transcription factors or recruiting methyl-CpG binding proteins (MBDs) via histone methylation (22).

Methyl-CpG-binding domain (MBD) proteins, including MBD1/2/3/4/5/6 and MeCP2, are classified as epigenetic regulators (23). Recent studies have demonstrated the crucial functions of methyl- $\mathrm{CpG}$ binding proteins in epigenetic events in cancer (24), and MBD proteins were revealed to be involved in tumorigenesis as the initiators of DNA methylation (25). Among these proteins, MBD1 is the largest, and it has been demonstrated to be associated with cancer manifestation $(26,27)$. MBD1 plays a role in the drug resistance of cancers and immune cells (28). MBD1 evoked epigenetic changes via DNA methylation and the repressive H3K9me3 histone mark (29). Methylation of the lysine residue of histone $\mathrm{H} 3$ (H3K4) is highly associated with the transcriptional activation of genes, whereas methylation of $\mathrm{H} 3 \mathrm{~K} 9$ (H3K9me3) results in the recruiting of factors that inactivate transcription (30). Thus, these studies indicated that MBDs may act as a link between DNA methylation and histone modification (31).

Although the roles of the TESC gene have been studied in cancer, the detailed molecular mechanism remains unclear. In addition, the involvement of TESC expression in the connection of DNA methylation and histone methylation via MBD1 is largely unknown. Therefore, the aim of the present study was to investigate the role of TESC expression in epigenetic regulation. Our data revealed that DNA methylation was negatively associated with TESC gene expression in gastric cancer cells. Moreover, both DNA methylation and histone modification via MBD1 may play potential roles in the regulation of TESC expression.

\section{Materials and methods}

Cell lines and culture conditions. The human gastric cancer cell lines, AGS, SNU-216, NCI-N87, SNU-620, SNU-638,
NUGC-3, and MKN-74; human colon cancer cell lines, COLO205, DLD-1, HCT116, HT-29, KM12C, KM12SM, LS174T and SW480; human liver cancer cell lines, SK-Hep-1 and Huh-7; human cervical cancer cell lines, CaSki and SiHa; human lung cancer cell line, A549; and human breast cancer cell line, MCF-7, were purchased from the American Type Culture Collection (ATCC; Manassas, VA, USA) and grown in Dulbecco's modified Eagle's medium (DMEM) supplemented with $10 \%$ fetal bovine serum (FBS). Cells were grown at $37^{\circ} \mathrm{C}$ in a humidified, $5 \% \mathrm{CO}_{2} /$ air atmosphere. To identify regulation by methyltransferase, 5-aza-2'-deoxycytidine (1 $\mu \mathrm{M}$; Sigma-Aldrich; Merck KGaA, Darmstadt, Germany), a methyltransferase inhibitor, was added to the culture medium for $72 \mathrm{~h}$ to induce demethylation of the cytosine residues.

Sodium bisulfite modification and methylation-specific PCR (MSP). Chromosomal DNA was isolated from the cell cultures grown in $100 \mathrm{~mm}$ cell culture dishes (Corning Inc., Corning, NY, USA) using a genomic DNA purification kit (Promega Corp., Madison, WI, USA) according to the manufacturer's protocol. The extracted DNA was eluted with $250 \mu \mathrm{l}$ of distilled water. Sodium bisulfite modification of genomic DNA was carried out using an EZ DNA Methylation kit (Zymo Research Corp., Irvine, CA, USA) according to the manufacturer's protocol using $0.1 \mathrm{mg}$ of purified DNA. PCR was carried out as described previously using primers (Table SI) and a Power SYBR-Green kit (Applied Biosystems; Thermo Fisher Scientific,Inc., Waltham,MA,USA) (20). The methylation index was calculated for each sample using the following formula: Methylation index $=[1 /(1+2-(\mathrm{CTu}-\mathrm{CTme})] \mathrm{x} 100 \%$, where $\mathrm{CTu}$ was the average cycle threshold (CT) obtained from duplicate quantitative PCR analyses using the unmethylated primer pair and CTme was the average CT obtained using the methylated primer pair.

Cell viability assay. Cell viability was assessed with WST-1 assays (Roche Diagnostics GmbH, Mannheim, Germany) according to the manufacturer's protocol. Briefly, $10 \mu \mathrm{l}$ of WST-1 reagent was added to each well of a 96-well plate $\left(1 \times 10^{3}\right.$ cells/well). After incubation for $1 \mathrm{~h}$, the conversion of the WST-1 reagent into chromogenic formazan was evaluated with a spectrophotometer (Molecular Devices, LLC, Sunnyvale, CA, USA).

RT-PCR and real-time PCR. Total RNA from cells cultured in a 100-mm cell culture dish (Corning Inc.) was prepared using TRIzol reagent (Invitrogen; Thermo Fisher Scientific, Inc.) according to the manufacturer's protocols. Reverse transcription was conducted using $10 \mu \mathrm{g}$ of total RNA with a reverse transcription kit (Promega Corp.). The expression levels of the studied genes were measured by RT-PCR and real-time PCR analysis. One microliter of cDNA was used for the PCR, and triplicate reactions were performed for each sample using an AccuPower PCR premix (Bioneer Corp., Daejeon, Korea) and a Power SYBR-Green kit (Applied Biosystems; Thermo Fisher Scientific, Inc.) with gene-specific primers on an ABI ProFlex PCR system (Applied Biosystems; Thermo Fisher Scientific, Inc.) and an ABI StepOnePlus instrument (Applied Biosystems; Thermo Fisher Scientific, Inc.). The primers used for this selected gene are listed in Table SI. 
RNA quantity was normalized to that of $\beta$-actin or GAPDH, and gene expression was quantified according to the $2-\Delta \mathrm{Cq}$ method (32).

Western blot analysis. Cells cultured in a $100-\mathrm{mm}$ cell culture dish (Corning Inc.) were resuspended in RIPA lysis buffer [50 mmol/l Tris- $\mathrm{HCl}, \mathrm{pH} 7.4,150 \mathrm{mmol} / \mathrm{l} \mathrm{NaCl}, 1 \%$ NP40, $0.25 \%$ Na-deoxycholate, $1 \mathrm{mmol} / 1$ phenylmethylsulfonyl fluoride (PMSF), $1 \mathrm{mmol} / 1$ sodium orthovanadate, $1 \mathrm{X}$ protease inhibitor cocktail] (Sigma-Aldrich; Merck KGaA). Protein was assessed using a BCA Protein Assay Kit (Thermo Fisher Scientific, Inc.). Proteins were size-fractionated by $12 \%$ SDS-PAGE and transferred to a polyvinylidene difluoride (PVDF) membrane (Millipore Corp., Billerica, MA, USA). Non-specific binding was blocked by incubation with phosphate-buffered saline with Tween-20 (PBST) with 5\% powdered milk and $1 \%$ Triton X-100. Membranes were incubated overnight at $4^{\circ} \mathrm{C}$ with primary antibodies (TESC; dilution 1:1,000; cat. no. 11125-1-AP; ProteinTech Group, Inc., Chicago, IL, USA), (MBD1, Sp-1 and MeCP2; dilution 1:1,000; cat nos. SC-55473, SC-420 and SC-20700; Santa Cruz Biotechnology, Inc., Dallas, TX, USA), (H3K4me1, H3K4me2, H3K4me3, H3K 9me3, H3K27me3 and H3K36me3; dilution 1:1,000; cat. nos. ab8895, ab7766, ab8580, ab8898, ab6147 and ab9050, respectively; Abcam, Cambridge, MA, USA) followed by incubation with polyclonal HRP-conjugated secondary antibodies (dilution 1:2,000; cat. nos. a6667 and a9917; Sigma-Aldrich; Merck KGaA) for $1 \mathrm{~h}$ at room temperature. The membranes were incubated in Clarity Western ECL Substrate (Bio-Rad Laboratories, Inc., Hercules, CA, USA) and the protein bands were visualized by exposure to X-ray film. Protein loading was visualized by incubation of stripped membranes with a monoclonal antibody to $\beta$-actin (dilution 1:1,000; cat. no. SC-47778; Santa Cruz Biotechnology, Inc.).

Cell transfection. SNU-638 and NCI-N87 cells $\left(1 \times 10^{5}\right.$ cell/well) grown in 24-well plates (Corning Inc.) were transfected with specific-siRNAs ( $30 \mathrm{nmol} / \mathrm{ml}$ ) targeting TESC (Bioneer Corp.) and the pCMV-sports6-TESC vector $(2 \mu \mathrm{g} / \mu \mathrm{l})$ for $24 \mathrm{~h}$ using Lipofectamine 2000 (Invitrogen; Thermo Fisher Scientific, Inc.) according to the manufacturer's protocols. The medium was replaced with DMEM containing 10\% FBS for $24 \mathrm{~h}$. After recovery, cell viability was evaluated with the WST-1 assay. The pCMV-sports6 control and pCMV-sports6 TESC vectors were obtained from the Korea Human Gene Bank (Medical Genomics Research Center, Korea Institute of Bioscience and Biotechnology, Daejeon, Korea).

Nuclear fractionation. Fractionation of nuclear extracts was carried out using a Nuclear Extract kit (Active Motif, Carlsbad, CA, USA) according to the manufacturer's instructions. Pellets were resuspended in $50 \mathrm{ml}$ of complete lysis buffer, and supernatants were used as the nuclear fractions after centrifugation at $14,000 \mathrm{xg}$ for $10 \mathrm{~min}$ at $4^{\circ} \mathrm{C}$.

Chromatin immunoprecipitation (ChIP) assay. ChIP assays were carried out using an EZ ChIP Chromatin Immunoprecipitation kit (EMD Millipore, Billerica, MA, USA) as described in the supplier's protocol. Briefly, the cross-linked chromatin was sonicated after cell lysis and then incubated with antibodies against MBD1, HDAC2, Oct-1 and Sp-1 (dilution 1:1,000; cat. nos. SC-55473, SC-7899, SC-232 and SC-420, respectively; Santa Cruz Biotechnology, Inc.) at $4^{\circ} \mathrm{C}$ overnight. The immunocomplex was precipitated with Protein A-agarose (EMD Millipore), and the beads were washed, sequentially treated with $10 \mu 1$ of RNase A $\left(37^{\circ} \mathrm{C}\right.$ for $\left.30 \mathrm{~min}\right)$ and $75 \mu \mathrm{l}$ of Proteinase $\mathrm{K}\left(45^{\circ} \mathrm{C}\right.$ for $\left.4 \mathrm{~h}\right)$, and incubated at $65^{\circ} \mathrm{C}$ overnight to reverse cross-link the chromatin. The DNA was recovered by phenol-chloroform extraction and co-precipitation with glycogen and dissolved in $50 \mu 1$ of Tris-EDTA (TE) buffer. Extracted DNA was amplified by PCR using $1 \mu 1$ of the precipitated DNA. PCR primers (sequences are presented in Table SI) were designed to amplify the expected Oct-1 or Sp-1 binding sites at the TESC gene promoter. The real-time PCR conditions were 40 cycles at $94^{\circ} \mathrm{C}$ for $40 \mathrm{sec}, 60^{\circ} \mathrm{C}$ for $1 \mathrm{~min}$, and $72^{\circ} \mathrm{C}$ for $40 \mathrm{sec}$.

Statistical analysis. The one-way analysis of variance (ANOVA) with the Tukey's post hoc test and the Student's t-test were used to detect differences in the methylation and expression levels in low- and high-TESC-expressing gastric cancer cell lines using SPSS for Windows, release 17.0 (SPSS, Inc., Chicago, IL, USA). P-values $<0.05$ were considered to indicate a statistically significant difference.

\section{Results}

Differential expression of TESC $m R N A$ and protein in gastric cancer cell lines. To profile TESC gene expression in gastric cancer, we examined TESC mRNA and protein expression status in gastric cancer cell lines (Fig. 1A and B). RT-PCR revealed the upregulation of TESC mRNA expression in the SNU-620 cell line and a moderate level of TESC mRNA in the AGS, SNU-216, and NCI-N87 cell lines. Conversely, the TESC level was significantly downregulated in the SNU-638, NUGC-3 and MKN-74 cell lines. The results of quantitative real-time PCR also supported this observation (Fig. 1C). Moreover, the protein level of TESC also exhibited differential expression in the gastric cancer cell lines (Fig. 1B). Collectively, our results indicated that the TESC gene was differentially expressed in cancer cell lines. Given the recent studies indicating that epigenetic regulatory mechanisms such as promoter methylation are one of the vital mechanisms for the regulation of gene expression $(33,34)$, these results may indicate that the hypermethylation of the promoter inactivates the expression of the TESC gene.

Aberrant hypermethylation of the TESC gene promoter in gastric cancer cell lines. The methylation level of the TESC gene in various $\mathrm{CpG}$ sites of the proximal promoter using the NCBI GEO database (www.ncbi.nlm.nih.gov/geo, datasets for GSE25869) was then analyzed and the significant hypermethylation of the TESC promoter was revealed in various normal tissues compared with that in cancer tissues, including colon, breast, brain, lung and gastric cancers (data not shown). It was thus hypothesized that promoter methylation of the TESC gene was a key mechanism for regulating TESC expression. $\operatorname{cg} 06750167$ (probe ID) of $\mathrm{CpG}$ sites on the TESC gene promoter revealed very different methylation 
A

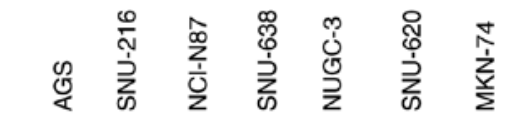

TESC

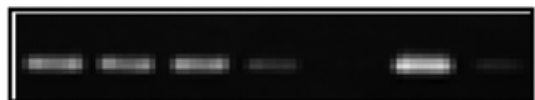

$\beta$-actin

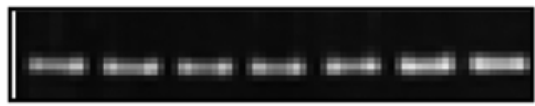

B

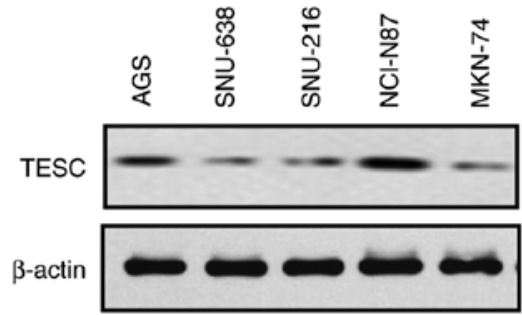

C

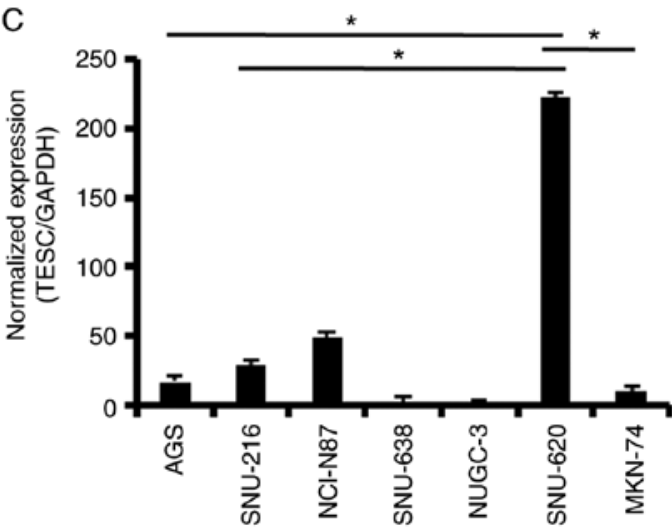

Figure 1. TESC expression in gastric cancer cell lines. (A and C) mRNA expression of TESC was examined by RT-PCR and real-time PCR in gastric cancer cell lines. (B) Protein levels were evaluated by western blotting in gastric cancer cell lines. $\beta$-Actin or GAPDH was selected as an internal control Each sample was examined in three independent reactions, and the average relative level is presented with the standard error; ${ }^{*} \mathrm{P}<0.05$. TESC, tescalcin.

levels in gastric cancer compared with those in normal tissues (Fig. 2A). To confirm the differential methylation and its expression level, real-time MSP (methylation-specific PCR) was performed in gastric cancer cell lines to analyze the specific $\mathrm{CpG}$ site methylation status in gastric cancer cell lines. As revealed in Fig. 2B, a significant amplification was detected in gastric cancer cell lines, including AGS, SNU-216, NCI-N87, SNU-638 and NUGC-3 cells, by comparing the methylation levels of the methylation-specific signal with the unmethylation-specific signal (Fig. 2B). Thus, these results revealed that the CpG site of the TESC promoter was likely to be hypermethylated in gastric cancer cell lines, which may suggest that the increased methylation level of the cg06750167 site was critical in determining the expression pattern of the TESC gene in gastric cancer.

Demethylation of the CPG site on the TESC promoter following treatment with 5-aza-dC in gastric cancer cell lines. Given the epigenetic regulation mechanism via the relationship between DNA methylation and opposite gene expression (33), and the finding that the TESC promoter was highly methylated in gastric cancer cell lines with the exception of SNU-620 cells,
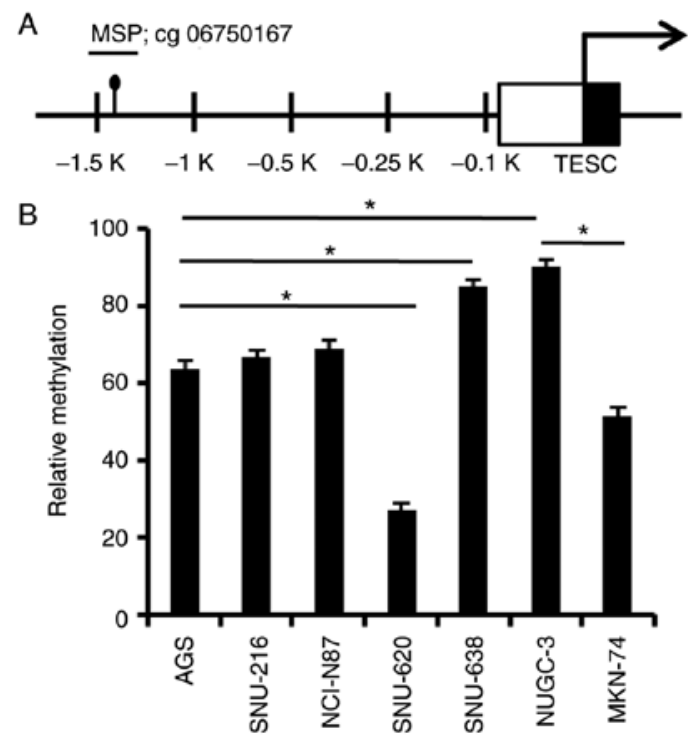

Figure 2. Methylation of the TESC gene promoter in gastric cancer cell lines. (A) Schematic diagram of the TESC promoter for MSP and real-time MSP. (B) Methylation of the TESC promoter was examined by real-time MSP in gastric cancer cell lines. Each sample was examined in three independent reactions, and the average relative level is presented with the standard error; ${ }^{*} \mathrm{P}<0.05$. TESC, tescalcin; MSP, methylation-specific PCR.

cell viability following 5 -aza-2c-deoxycytidine (5'-aza-dC) treatment was examined, however no obvious changes were observed, except in SNU-638 cells (Fig. 3A). Since the DNA methyltransferase (DNMT) inhibitor 5'-aza is able to cause DNA demethylation in the genome and induce the expression of silenced genes, additively, $1 \mu \mathrm{M}$ of aza is not an effective concentration to induce cell death in gastric cancer cell lines. Thus, the cell viability was not significantly altered in gastric cancer cell lines after AZA treatment. It is known that the epigenetic change in the methylation status of the specific CpG site is involved in transcription regulation (35). Thus, the promoter status after 5 '-aza-dC treatment was examined. To this end, 5-aza-dC, a methyltransferase inhibitor, was added to the gastric cancer cell lines, and methylation levels were evaluated by MSP and real-time MSP (Fig. 3B and C). As revealed in Fig. 3B, there was significant amplification with unmethylated primers in AGS, SNU-216, NCI-N87, SNU-638, NUGC-3 and MKN-74 cells following treatment with 5 '-aza-dC. This was further supported by the significant hypomethylation on the TESC promoter in gastric cancer cell lines treated with 5'-aza-dC (Fig. 3C). Numerical evaluation revealed 2.5-, 2.5-, 2.5-, 2.5-, 4- and 1-fold decreases in methylation on the TESC promoter following treatment with 5 '-aza-dC in AGS, SNU-216, NCI-N87, NUGC-3, MKN-74 and SNU-638 cells, respectively. Therefore, these findings indicated that 5 '-aza-dC-induced demethylation of the CpG site on the TESC promoter may be crucial in determining the expression of the TESC gene.

Demethylation of the TESC promoter by 5'-aza-dC leads to the expression of TESC in gastric cancer cell lines. Given that the treatment with 5'-aza-dC depleted promoter methylation, it was examined whether promoter hypomethylation induced by 5 '-aza-dC $(1 \mu \mathrm{M})$ treatment for 3 days 

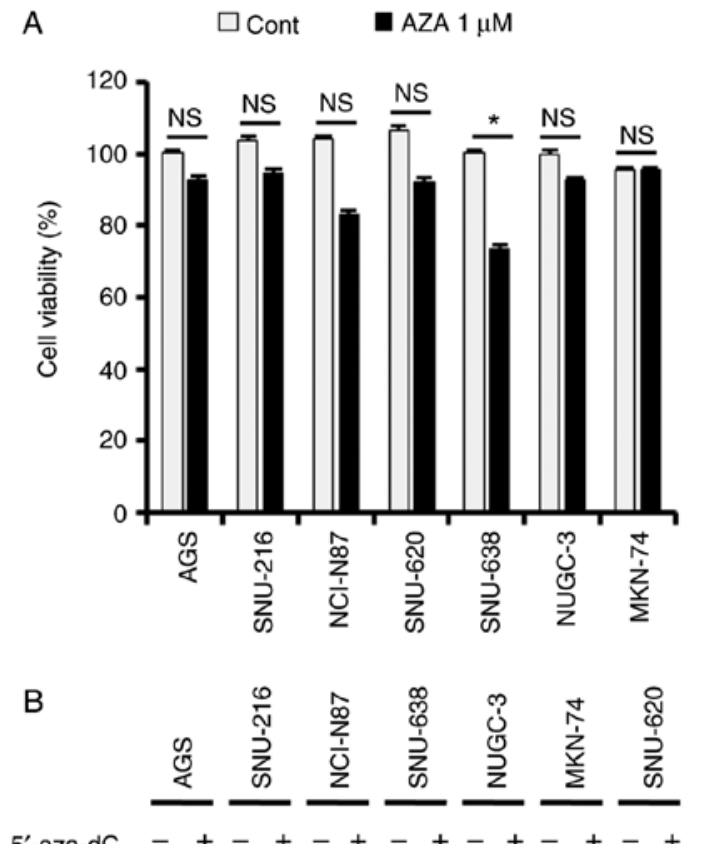

5'aza-dC -++-++-++-+

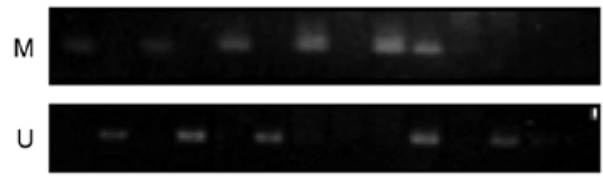

C

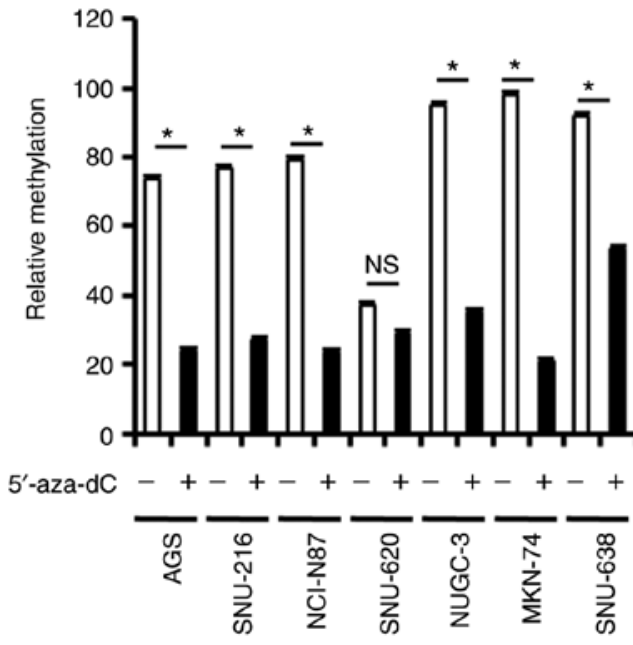

Figure 3. 5'-Aza-dC induces the demethylation of the CpG site on the TESC promoter in gastric cancer cell lines. After gastric cancer cell lines were treated with 5'-aza-dC, (A) cell viability was assessed by WST-1, and the methylation level was analyzed by (B) MSP and (C) real-time MSP. Each sample was examined in three independent reactions, and the average relative level is presented with the standard error; ${ }^{2} \mathrm{P}<0.05$; n.s, not significant; 5'-aza-dC, 5-aza-2c-deoxycytidine; TESC, tescalcin; MSP, methylation-specific PCR.

led to the expression of the TESC gene. The RT-PCR and real-time PCR results indicated the upregulation of TESC mRNA following 5 '-aza-dC treatment in some gastric and colorectal cancer cell lines (Figs. 4A and B, and 5A and B). Quantitative real-time PCR data also revealed that 5'-aza-dC treatment increased TESC expression by 5-, 3-, 0.5-, 6- and 0.7 -fold in the AGS, SNU-216, NCI-N87, SNU-638 and SNU-620 gastric cancer cell lines, respectively, and there were 0.5-, 2- and 0.8-fold increases in the HT-29, KM12C,
A
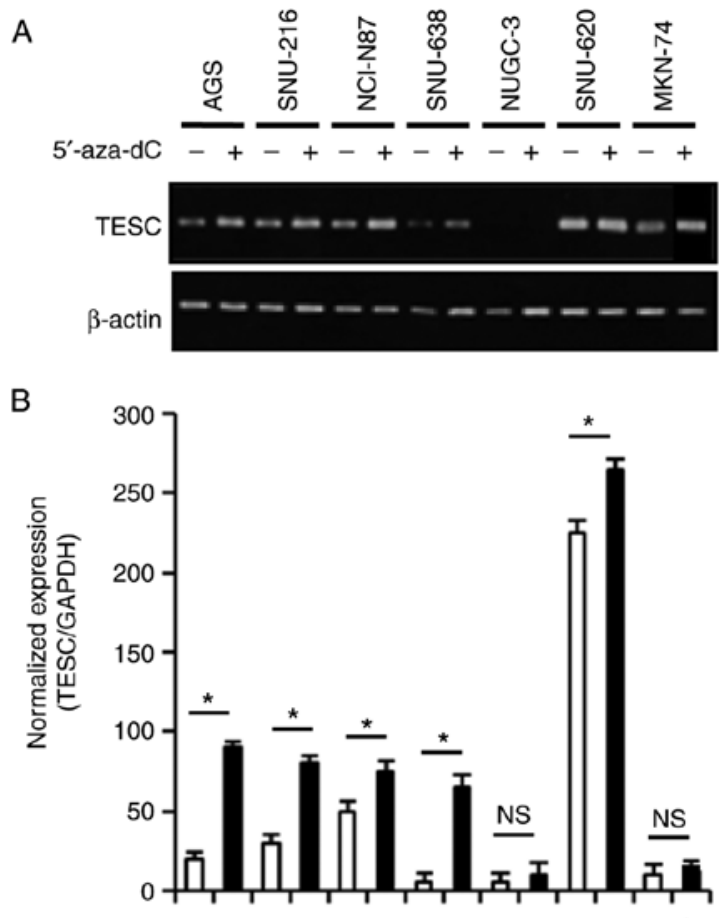

$5^{\prime}$-aza-dC

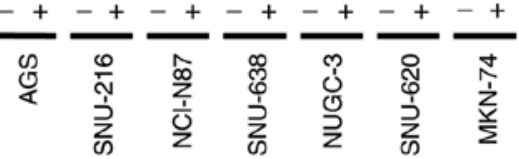

C

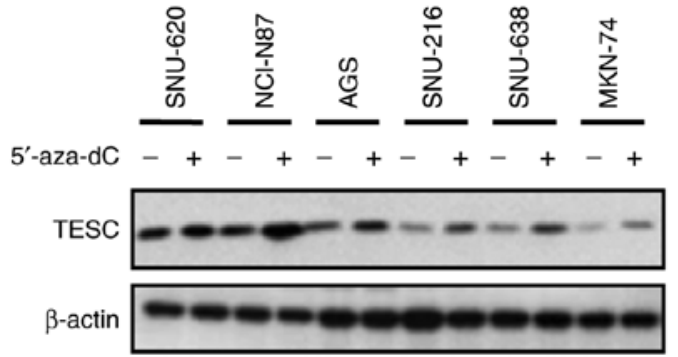

Figure 4. 5'-Aza-dC induces the upregulation of TESC in gastric cancer cell lines. After gastric cancer cell lines were treated with 5'-aza-dC, the mRNA level of TESC was assessed by (A) RT-PCR and (B) quantitative real-time PCR. (C) The protein levels of TESC were analyzed by western blotting. $\beta$-Actin or GAPDH was selected as an internal control. Each sample was examined in three independent reactions, and the average relative level is presented with the standard error; ${ }^{\prime} \mathrm{P}<0.05$; n.s, not significant; 5 '-aza-dC, 5-aza-2c-deoxycytidine; TESC, tescalcin; MSP, methylation-specific PCR.

and KM12SM (Figs. 4B and 5B) colorectal cancer cell lines, respectively. In agreement with the TESC expression results, the protein levels in 5-aza-dC-treated gastric cancer cell lines were upregulated after 5'-aza-dC treatment (Fig. 4C). In the MKN-74 cell line, the expression of TESC was weakly increased after 5'-aza-dC treatment as shown in Fig. 4A and C. The results revealed that the response of MKN-74 cells after 5'-aza-dC treatment was not different compared to the responses of other gastric cancer cell lines. Overall, these data indicated that the 5'-aza-dC-induced hypomethylation in the TESC promoter restored TESC expression. These results strongly indicated that TESC gene expression was associated with the methylation status of a specific $\mathrm{CpG}$ site, and the methylation level of this $\mathrm{CpG}$ site was one of the key factors for TESC mRNA and protein expression in gastric cancer cell 
A

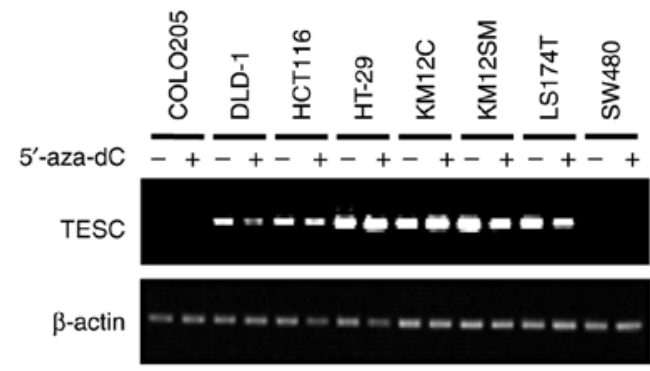

B

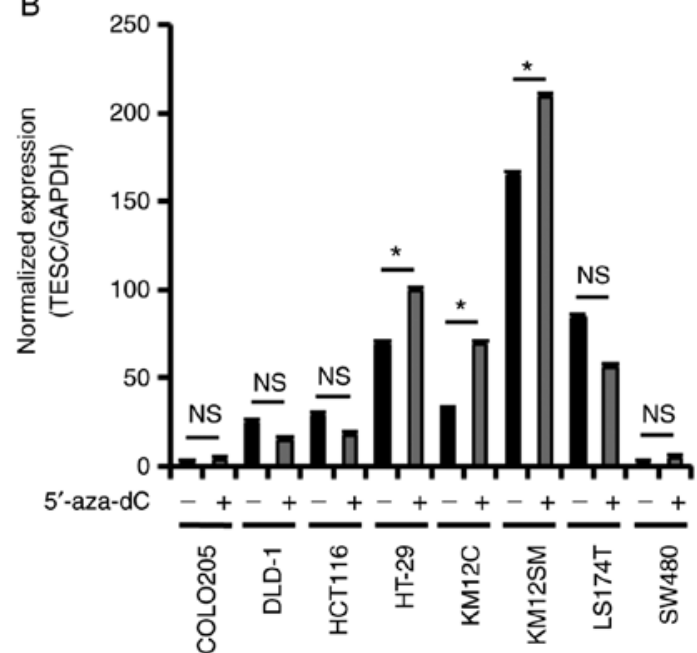

Figure 5. 5'-Aza-dC does not significantly alter TESC expression in colorectal cancer cell lines. After colorectal cancer cell lines were treated with 5'-aza-dC, the mRNA level of TESC was assessed by (A) RT-PCR and (B) real-time PCR. $\beta$-Actin or GAPDH was selected as an internal control. Each sample was examined in three independent reactions, and the average relative level is presented with the standard error; ${ }^{*} \mathrm{P}<0.05$; n.s, not significant; 5'-aza-dC, 5-aza-2c-deoxycytidine; TESC, tescalcin.

lines. Therefore, the transcriptional and translational regulation mechanism that regulates TESC gene expression via DNA methylation was identified.

Epigenetic modification of the TESC gene is mediated by MBD1 and Oct-1 via HDAC2 in gastric cancer cell lines. It has been reported that the accumulation of the MBD protein associated with the 5'-methylated cytosine residue is involved in the transcriptional regulation of promoter methylation (36). MBD proteins form a complex with corepressors, including HDACs and Sin3A, and facilitate the assembly of a repressive chromatin structure $(37,38)$. Another silencing mechanism via DNA methylation is the binding of transcription factors such as Sp-1 and Oct-1 at gene promoters (39-41). A recent study revealed that the mouse TESC gene contained $\mathrm{CpG}$ islands on the promoter region and putative transcription factor binding sites for Sp-1, EGFR1, ZBP-89, AP-2 and CDF-1 (42).

To determine whether methylation of the $\mathrm{CpG}$ site at the TESC promoter influences the binding of candidate transcription factors, the promoter region was divided into four parts (R1-R4), ranging from -0.1 to $-1.5 \mathrm{~K}$, to identify the localization of the transcription factors (Fig. 6A). To assess the chromatin localization of the putative regulators such as MBD1, HDAC2, Oct-1 and Sp-1, ChIP was performed with relevant antibodies in hypermethylated AGS and SNU-638 cells to assess the chromatin localization of the regulators (Fig. 6B and C).
The binding of the epigenetic transcription repressor complex MBD1 and HDAC2 was only observed in the R4 region, which contains the differential CpG methylation site. The binding of MBD1 and HDAC2 was observed in TESC downregulated AGS and SNU-638 cells, but not in 5'-aza-dC-treated AGS and SNU-638 cells (Fig. 6B and C). Furthermore, the ChIP analysis of the transcription factors Oct- 1 and $\mathrm{Sp}-1$ at the R4 region revealed the significant localization of Oct-1 in AGS and SNU-638 cells with a hypermethylated TESC promoter. The Oct-1 localization was reduced following treatment with 5'-aza-dC (Fig. 6B and C). Collectively, these findings indicated that in AGS and SNU-638 cells, the binding of MBD1 to the methylated $\mathrm{CpG}$ site of the TESC promoter resulted in the recruitment of corepressors, such as HDAC2, and that the binding of Oct-1 was also critical for the transcriptional inhibition of TESC by binding to MBD1 and HDAC2.

TESC negatively regulates MBDI and histone methylation and enhances cell survival in gastric cancer. Given the aforementioned results, whether TESC loss negatively regulated MBD1 expression was determined, and for this, a small interfering RNA against TESC (siTESC) and was constructed and used to treat NCI-N87 cells, which exhibited high TESC expression levels. Fig. 7C revealed the significant downregulation of TESC expression in NCI-N87 cells that were treated with siTESC compared with that in cells transfected with siCont (Fig. 7C). In addition, the WST-1 assay results indicated that siTESC reduced the viability of NCI-N87 cells by $30 \%$ compared to that in the controls (Fig. 7A). Notably, the upregulation of MBD1 following siTESC treatment and the downregulation of $\mathrm{H} 3 \mathrm{~K} 4 \mathrm{me} 2$ and $\mathrm{H} 3 \mathrm{~K} 4 \mathrm{me} 3$, which are active transcription markers was detected. However, no obvious change was observed for other histone methylations such as H3K4me1 and H3K9me3 (Fig. 7C). Thus, our results indicated that the silencing of TESC by promoter hypermethylation suppressed the survival of gastric cancer cells (Fig. 8A).

Considering the result that TESC loss suppresses the survival of gastric cancer cells, TESC was overexpressed using the p-CMV-sports6-TESC vector in SNU-638 cells, which express a low level of TESC. Fig. 7B revealed that overexpression of TESC increased cell proliferation by $10 \%$ (Fig. 7B). In addition, western blot analysis demonstrated that TESC overexpression significantly inhibited MBD1 expression, while the levels of $\mathrm{H} 3 \mathrm{~K} 4 \mathrm{me} 2$ and $\mathrm{H} 3 \mathrm{~K} 4 \mathrm{me} 3$ increased (Fig. 7D). Collectively, our findings provided evidence that TESC upregulated $\mathrm{H} 3 \mathrm{~K} 4 \mathrm{me} 2$ and $\mathrm{H} 3 \mathrm{~K} 4 \mathrm{me} 3$ for transcriptional activation and thus indicated that overexpression of TESC by promoter hypomethylation promoted cell survival in gastric cancer (Fig. 8B).

\section{Discussion}

The present study demonstrated that TESC expression was negatively regulated by promoter methylation via MBD1 and that its overexpression promoted the survival of gastric cancer cells, but not colorectal cancer cells. It has been reported the upregulation of TESC expression in radiation-induced thyroid cancer as well as acute myeloid leukemia resulted in acquired resistance against sorafenib via an interaction with NHE1 $(43,44)$. Previously, our research revealed that TESC 


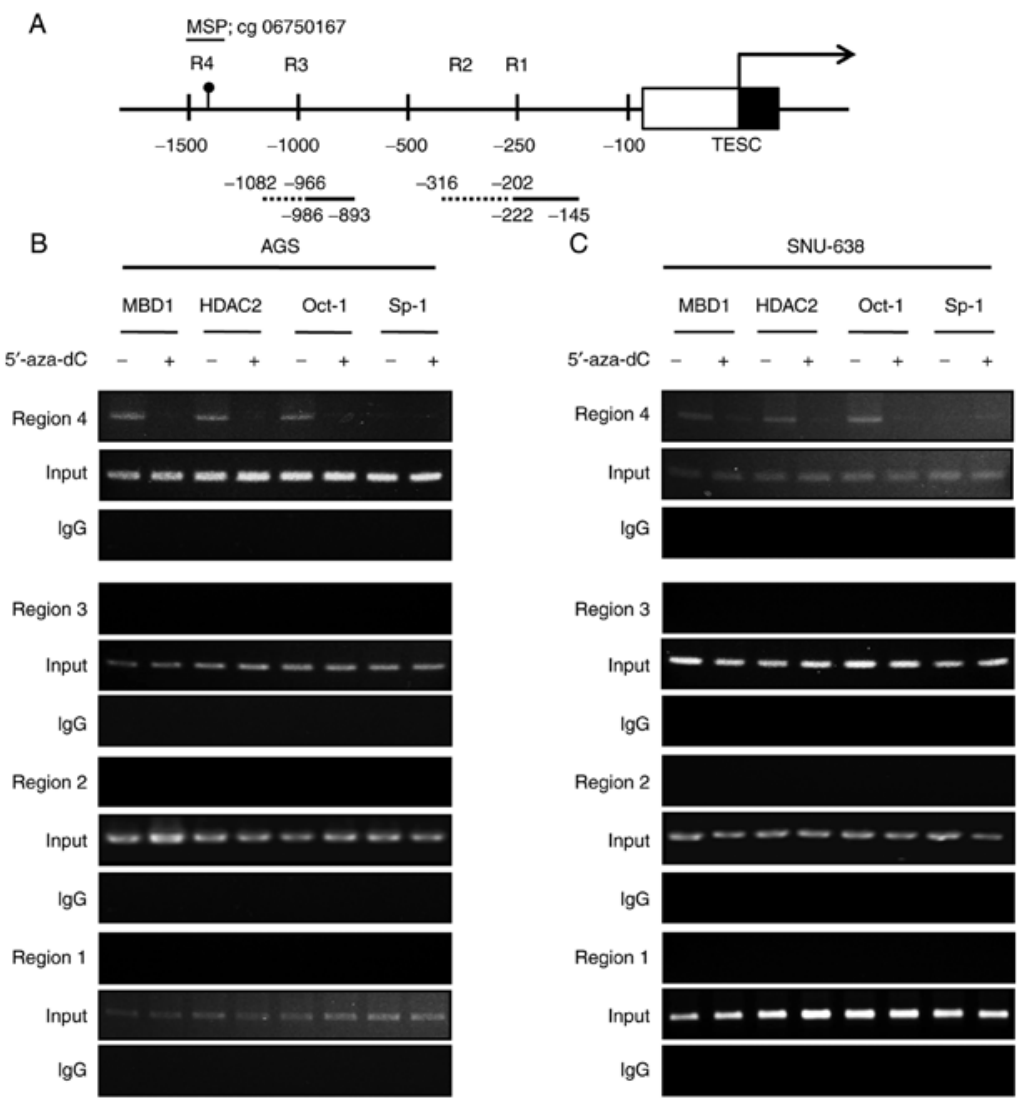

Figure 6. ChIP analysis of the TESC promoter against the candidates MBD1, HDAC2, Oct-1, and Sp-1. ChIP assays were conducted on the TESC promoter using anti-MBD1, anti-HDAC2, anti-Oct-1, and anti-Sp-1 by PCR to amplify the CpG site. (A) Schematic diagram of the TESC promoter indicating four candidate regions (R1-R4) for putative transcription factor binding. PCR analysis of the IP derived from DMSO- or 5'-aza-dC-mediated (B) AGS and (C) SNU-638 cells was performed using primers for the putative regions (R1-R4). Each sample was examined in three independent reactions. ChIP, chromatin immunoprecipitation; TESC, tescalcin; MBD, methyl-CpG binding domain protein; HDAC, histone deacetylase; IP, immunoprecipitated DNA; DMSO, dimethyl sulphoxide.

A

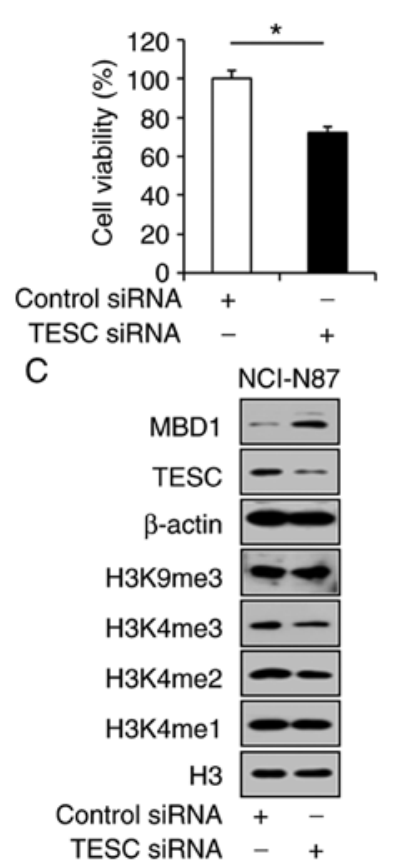

B

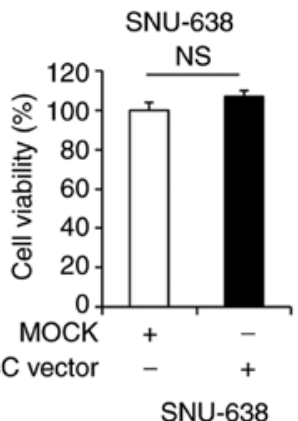

D

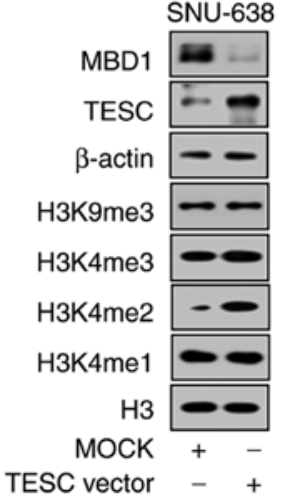

Figure 7. Knockdown and overexpression of TESC regulate MBD1 and histone methylation. NCI-N87 cells were transfected with control siRNA or siRNA targeting TESC and treated with 5'-aza-dC. SNU638 cells transfected with the pCMV-sports6 vector and TESC vector and with 5'-aza-dC. (A and B) Cell viability assays were conducted in TESC siRNA-transfected NCI-N87 and vector for TESC-transfected SNU638 cells, respectively. (C and D) Western blot analysis of MBD1 and TESC used proteins extracted from whole cell lysates, and histone methylation analysis was performed using the nuclear fraction. $\beta$-Actin or $\mathrm{H} 3$ was selected as an internal control. Each sample was examined in three independent reactions, and the average relative level is presented with the standard error; ${ }^{*} \mathrm{P}<0.05 ;$ n.s, not significant; TESC, tescalcin; MBD, methyl-CpG binding domain protein. 

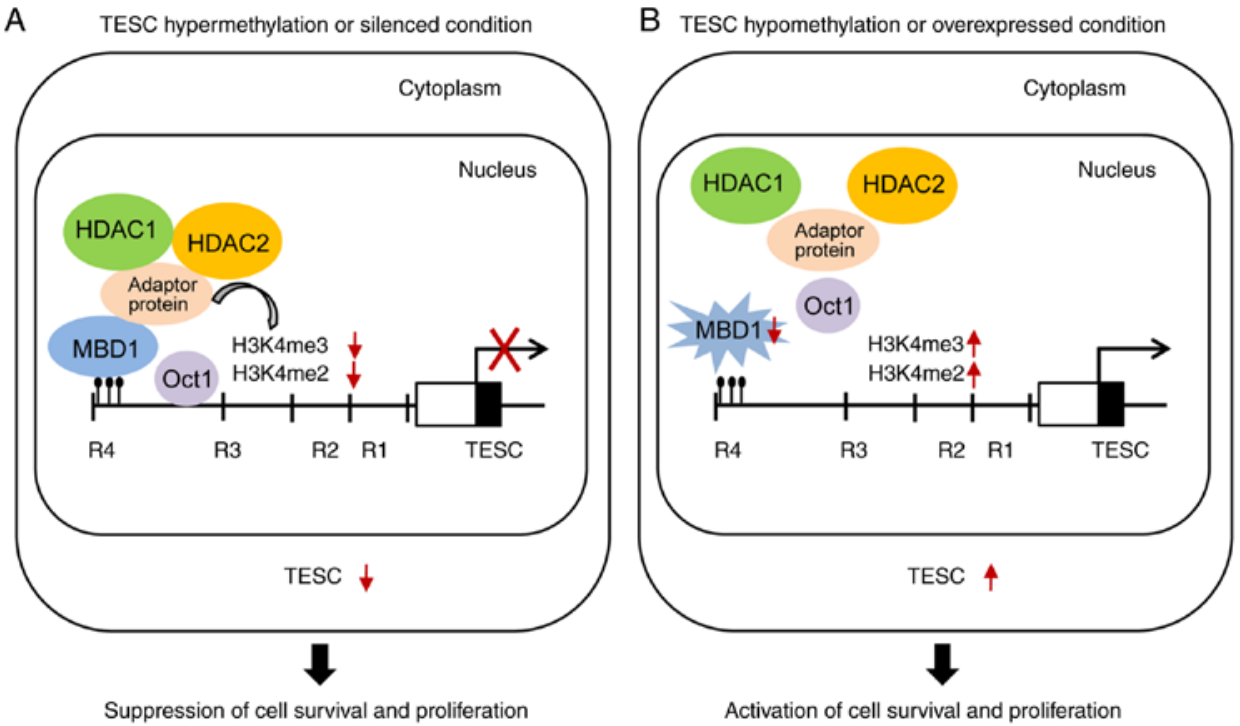

Figure 8. The proposed molecular mechanism in which (A) TESC inhibition results in the induction of hypermethylation of the promoter to decrease cell survival in gastric cancer and (B) overexpression of TESC enhances promoter hypomethylation to increase cell survival. TESC, tescalcin.

expression was upregulated in colorectal cancer tissues compared with that in normal tissues and that the inhibition of TESC blocked NF-kB signaling and decreased cell survival in vitro and in vivo (6). Furthermore, the upregulation of TESC expression promoted migration and invasion via the activation of epithelial-mesenchymal transition (EMT) in colorectal cancer cells (7). Overall, these studies suggested that the identification of the regulatory mechanism of TESC expression could provide effective therapeutic strategies for diverse types of cancer.

In the present study, the differential expression of TESC in gastric cancer cell lines was observed; TESC was upregulated in AGS, SNU-216, NCI-N87, and SNU-620 cells but was downregulated in SNU-638, NUGC-3 and MKN-74 cells. However, comparison to a normal gastric epithelial cell line could not be performed since we could not obtain a normal gastric cell line and the ATCC does not carry such a cell line. No association between methylation and malignant status was discovered. Notably, TESC expression was negatively regulated via epigenetic regulation such as DNA methylation and histone modification (33). DNA methylation is considered one of the most powerful epigenetic modifications (34) and plays important roles in silencing of tumor suppressor genes via hypermethylation and activating of oncogenes via hypomethylation in cancer (45). To identify specific demethylated genes in the NCBI Dataset, 32 pairs of human normal gastric and cancer tissues were analyzed to discover specific methylation signatures using microarray with the Illumina HumanMethylation27 BeadChip (Human Methylation27_270596_v.1.2). Furthermore, it was revealed that TESC expression was negatively regulated by promoter methylation in gastric cancer cells, not colorectal cancer cells. Real-time MSP revealed the hypermethylation of the TESC promoter in gastric cancer cells, except for SNU-620 cells. In addition, its hypermethylation was confirmed only in HT-29 and KM12C colorectal cancer cells. Consistent with these results, when cells were treated with 5'-aza-dC, a methyltransferase inhibitor, real-time MSP/RT-PCR and western blot analyses indicated the critical role that the methylation level of the promoter $\operatorname{cg} 06750167$ site in the TESC gene plays in its expression. Accumulating evidence has indicated that promoter methylation was involved in the transcription regulatory mechanism of cancer-related genes, such as MTO1, MRPL41, TCF21 and ZNF331 (46-48). Moreover, additional evidence has suggested that $\mathrm{CpG}$ sites in the TESC promoter caused different methylation patterns in cold blood from 336 Mexican-American newborns after prenatal phthalate exposure (49). Additionally, patients with major depressive disorder were revealed to have hypermethylation of $\mathrm{CpG}$ sites on a TESC gene-regulating genetic variant (rs7294919) (50). In addition, histone deacetylases (HDACs) play crucial roles in epigenetic regulation of protein expression (51). HDAC inhibition also regulates processes such as DNA histone methylation and gene expression via DNMT1 regulation (52). This was supported by the finding that treatment with Class I HDAC inhibitors upregulated the TESC gene in neurons and had a neuroprotective effect via an epigenetic mechanism (1).

Several researchers have reported the epigenetic mechanisms through the regulation of genes by DNA methylation via transcription factors such as E2F, Sp-1, Oct-1 and CREB, or via methyl-CpG-binding proteins such as MBD1, MBD2, MBD3 and MeCp2 (22,53-57). Notably, the present results demonstrated that TESC expression was suppressed by the binding of MBD1, HDAC2, and Oct-1 to its promoter; conversely, 5'-aza-dC treatment restored TESC expression by blocking MBD1, HDAC2, and Oct-1 binding on putative methylation sites. In support of these data, putative $\mathrm{Sp}-1$ and Sp3 binding on the promoter of the mouse TESC gene was reported by Perera et al (41). However, Sp-1 binding was not revealed on the TESC promoter in DMSO- or 5'-aza-dC-induced gastric cancer cells. MBD1, a transcriptional regulator, has been shown to repress the transcription of tumor suppressor genes, such as p16 and VHL, by binding their CpG sites (58). It has been reported that the binding of MBDs and HDACs (HDAC1 and HDAC2) to promoters plays a crucial role in the suppression of protein expression (59). In line with 
this, the present study demonstrated that vital regulators, including MBD1, HDAC2 and Oct-1, via CpG methylation were involved in the regulation of TESC expression and that 5'-aza-dC blocked these repressors by demethylation.

MBD proteins are considered critical regulators of gene regulation via chromatin modification (60). Moreover, the MBD proteins have been demonstrated to bind DNAs containing methylated $\mathrm{CpG}$ sites (61). To investigate the effects of the binding of mono-, di-, and trimethylated histone $\mathrm{H} 3 \mathrm{~K} 4$ and trimethylated histone $\mathrm{H} 3 \mathrm{~K} 9$ on the regulation of TESC expression, histone methylation status was examined in siTESC-transfected NCI-N87 cells and in TESC vector-transfected SNU-638 cells. Histone methylation analysis revealed that differential TESC expression was associated with MBD1 and histone methylation. Suppression of TESC upregulated MBD1 expression and conversely downregulated $\mathrm{H} 3 \mathrm{~K} 4 \mathrm{me} 3$ and decreased cell viability. In addition, loss of $\mathrm{H} 3 \mathrm{~K} 4 \mathrm{me} 2 / 3$ caused cell death, whereas high expression of $\mathrm{H} 3 \mathrm{~K} 4 \mathrm{me} 2 / 3$ induced cell survival, resulting in increased migration and invasion (62-64). In these findings, loss of TESC mediated the increase in MBD1 and decrease in $\mathrm{H} 3 \mathrm{~K} 4 \mathrm{me} 2 / 3$ and then induced cell death. In contrast, overexpression of TESC using a TESC expression vector decreased MBD1 levels and enhanced the expression of H3K4me2/3. Previously, our team of researchers reported that TESC expression was involved in cell migration, invasion and EMT in colorectal cancer, but that depletion of TESC induced cell death (6,7). Based on our findings, we propose a detailed mechanism for the regulation of TESC expression in which TESC expression promotes cell survival for invasion and migration via DNA hypomethylation, decreases MBD1, and increases $\mathrm{H} 3 \mathrm{~K} 4 \mathrm{me} 2 / 3$, whereas loss of TESC may induce cell death via promoter hypermethylation, accumulation of MBD1, and inhibition of $\mathrm{H} 3 \mathrm{~K} 4 \mathrm{me} 2 / 3$. In a future study, we may analyze the methylation status of TESC promoter using tissues of patients with gastric cancer compared to normal tissues and define the relationships between TESC methylation and gastric cancer.

In conclusion, TESC expression was regulated by the methylation status of the promoter in gastric cancer cell lines, indicating a relationship between TESC expression and its epigenetic modification, such as promoter methylation and histone methylation. Furthermore, 5'-aza-dC prevented the binding of MBD1, HDAC2, and Oct-1 to $\mathrm{CpG}$ sites in the TESC promoter. Moreover, knockdown of TESC inhibited $\mathrm{H} 3 \mathrm{~K} 4 \mathrm{me} 2 / 3$, which is a transcription active code, and decreased cell survival, whereas overexpression of TESC enhanced $\mathrm{H} 3 \mathrm{~K} 4 \mathrm{me} 2$ for transcriptional activation and increased cell survival. Collectively, our findings provided novel evidence for the epigenetic regulation of the $\mathrm{CpG}$ site of TESC, suggesting a mechanistic link between TESC expression and epigenetic regulation. These findings also indicated novel cancer therapeutic strategies based on this identified epigenetic regulatory mechanism in gastric cancer cells.

\section{Acknowledgements}

We would like to thank Dr Hogyu David Seo (Department of Biological Sciences, Korea Advanced Institute of Science and Technology), and Dr Soo Young Jun (Genome Research Center,
Korea Research Institute of Bioscience and Biotechnology) for editing help of this manuscript.

\section{Funding}

The present study was supported by the Basic Science Research Program and Mid-Career Researcher Program through the National Research Foundation of Korea (NRF) funded by the Korea government (2017R1D1A1B03031502, 2016R1A2B4007413 and 2017R1A2B2005629).

\section{Availability of data and materials}

The datasets used and/or analyzed during the present study are available from the corresponding author on reasonable request.

\section{Authors' contributions}

TWK, SRH, YHK and HGL designed the experiments and analyzed the data. TWK, SRH, JTK, SMY, MSL, SHL and YHK performed the experiments. TWK, SRH, YHK and HGL wrote the manuscript. YHK and HGL supervised the overall project. All authors read and approved the manuscript and agree to be accountable for all aspects of the research in ensuring that the accuracy or integrity of any part of the work are appropriately investigated and resolved.

\section{Ethics approval and consent to participate}

Not applicable.

\section{Patients consent for publication}

Not applicable.

\section{Competing interests}

The authors declare that they have no competing interests.

\section{References}

1. Takamatsu G, Katagiri C, Tomoyuki T, Shimizu-Okabe C, Nakamura W, Nakamura-Higa M, Hayakawa T, Wakabayashi S, Kondo T, Takayama C, et al: Tescalcin is a potential target of class Ihistone deacetylase inhibitors in neurons. Biochem Biophys Res Commun 482: 1327-1333, 2017.

2. Mailänder J, Müller-Esterl W and Dedio J: Human homolog of mouse tescalcin associates with $\mathrm{Na}^{+} / \mathrm{H}^{+}$exchanger type-1. FEBS Lett 507: 331-335, 2001.

3. Malo ME and Fliegel L: Physiological role and regulation of the $\mathrm{Na}^{+} / \mathrm{H}^{+}$exchanger. Can J Physiol Pharmacol 84: 1081-1095, 2006.

4. Loo SY, Chang MK, Chua CS, Kumar AP, Pervaiz S and Clement MV: NHE-1: A promising target for novel anti-cancer therapeutics. Curr Pharm Des 18: 1372-1382, 2012.

5. Fan J, Xing Y, Wen X, Jia R, Ni H, He J, Ding X, Pan H, Qian G, $\mathrm{Ge} \mathrm{S}$, et al: Long non-coding RNA ROR decoys gene-specific histone methylation to promote tumorigenesis. Genome Biol 16: 139,2015

6. Kang YH, Han SR, Kim JT, Lee SJ, Yeom YI, Min JK, Lee CH, Kim JW, Yoon SR, Yoon DY, et al: The EF-hand calcium-binding protein tescalcin is a potential oncotarget in colorectal cancer. Oncotarget 5: 2149-2160, 2014.

7. Kang J, Kang YH, Oh BM, Uhm TG, Park SY, Kim TW, Han SR, Lee SJ, Lee Y and Lee HG: Tescalcin expression contributes to invasive and metastatic activity in colorectal cancer. Tumour Biol 37: 13843-13853, 2016. 
8. Levay $\mathrm{K}$ and Slepak VZ: Tescalcin is an essential factor in megakaryocytic differentiation associated with Ets family gene expression. J Clin Invest 117: 2672-2683, 2007.

9. Seth A, Ascione R, Fisher RJ, Mavrothalassitis GJ, Bhat NK and Papas TS: The ets gene family. Cell Growth Differ 3: 327-334, 1992.

10. Wasylyk B, Hahn SL and Giovane A: The Ets family of transcription factors. Eur J Biochem 211: 7-18, 1993.

11. Oikawa T and Yamada T: Molecular biology of the Ets family of transcription factors. Gene 303: 11-34, 2003.

12. Seth A and Watson DK: ETS transcription factors and their emerging roles in human cancer. Eur J Cancer 41: 2462-2478, 2005.

13. Hogart A, Lichtenberg J, Ajay SS, Anderson S; NIH Intramural Sequencing Center, Margulies EH and Bodine DM: Genome-wide DNA methylation profiles in hematopoietic stem and progenitor cells reveal overrepresentation of ETS transcription factor binding sites. Genome Res 22: 1407-1418, 2012.

14. Schones DE and Zhao K: Genome-wide approaches to studying chromatin modifications. Nat Rev Genet 9: 179-191, 2008.

15. Liu B, Tahk S, Yee KM, Fan G and Shuai K: The ligase PIAS restricts natural regulatory $\mathrm{T}$ cell differentiation by epigenetic repression. Science 330: 521-525, 2010.

16. Ling ZQ, Tanaka A, Li P, Nakayama T, Fujiyama Y, Hattori T and Sugihara $\mathrm{H}$ : Microsatellite instability with promoter methylation and silencing of hMLH1 can regionally occur during progression of gastric carcinoma. Cancer Lett 297: 244-251, 2010.

17. Qu Y, Dang S and Hou P: Gene methylation in gastric cancer. Clin Chim Acta 424: 53-65, 2013.

18. Yamamoto E, Suzuki H, Takamaru H, Yamamoto H, Toyota M and Shinomura Y: Role of DNA methylation in the development of diffuse-type gastric cancer. Digestion 83: 241-249, 2011.

19. Yu JS, Koujak S, Nagase S, Li CM, Su T, Wang X, Keniry M, Memeo L, Rojtman A, Mansukhani M, et al: $P C D H 8$, the human homolog of $P A P C$, is a candidate tumor suppressor of breast cancer. Oncogene 27: 4657-4665, 2008.

20. Ai L, Kim WJ, Kim TY, Fields CR, Massoll NA, Robertson KD and Brown KD: Epigenetic silencing of the tumor suppressor cystatin $\mathrm{M}$ occurs during breast cancer progression. Cancer Res 66: 7899-7909, 2006

21. Wolf I, O'Kelly J, Rubinek T, Tong M, Nguyen A, Lin BT, Tai HH, Karlan BY and Koeffler HP: 15-hydroxyprostaglandin dehydrogenase is a tumor suppressor of human breast cancer. Cancer Res 66: 7818-7823, 2006.

22. Hendrich B and Tweedie S: The methyl-CpG binding domain and the evolving role of DNA methylation in animals. Trends Genet 19: 269-277, 2003

23. Hendrich B and Bird A: Identification and characterization of a family of mammalian methyl-CpG binding proteins. Mol Cell Biol 18: 6538-6547, 1998.

24. Ballestar E and Esteller M: Methyl-CpG-binding proteins in cancer: Blaming the DNA methylation messenger. Biochem Cell Biol 83: 374-384, 2005.

25. Sansom OJ, Maddison K and Clarke AR: Mechanisms of disease Methyl-binding domain proteins as potential therapeutic targets in cancer. Nat Clin Pract Oncol 4: 305-315, 2007.

26. Jørgensen HF, Ben-Porath I and Bird AP: Mbd1 is recruited to both methylated and nonmethylated $\mathrm{CpG}$ via distinct DNA binding domains. Mol Cell Biol 24: 3387-3395, 2004.

27. Liu H, Jin G, Wang H, Wu W, Liu Y, Qian J, Fan W, Ma H, Miao R, $\mathrm{Hu} \mathrm{Z}$, et al: Methyl-CpG binding domain 1 gene polymorphisms and lung cancer risk in a Chinese population. Biomarkers 13 607-617,2008

28. Wang J, Tai LS, Tzang CH, Fong WF, Guan XY and Yang M: $1 \mathrm{p} 31,7 \mathrm{q} 21$ and $18 \mathrm{q} 21$ chromosomal aberrations and candidate genes in acquired vinblastine resistance of human cervical carcinoma KB cells. Oncol Rep 19: 1155-1164, 2008.

29. Monnier P, Martinet C, Pontis J, Stancheva I, Ait-Si-Ali S and Dandolo L: H19 lncRNA controls gene expression of the Imprinted Gene Network by recruiting MBD1. Proc Natl Acad Sci USA 110: 20693-20698, 2013.

30. Wang P, Lin C, Smith ER, Guo H, Sanderson BW, Wu M, Gogol M, Alexander T, Seidel C, Wiedemann LM, et al: Global analysis of H3K4 methylation defines MLL family member targets and points to a role for MLL1-mediated H3K4 methylation in the regulation of transcriptional initiation by RNA polymerase II. Mol Cell Biol 29: 6074-6085, 2009.

31. Zheng YG, Wu J, Chen Z and Goodman M: Chemical regulation of epigenetic modifications: Opportunities for new cancer therapy. Med Res Rev 28: 645-687, 2008.
32. Livak KJ and Schmittgen TD: Analysis of relative gene expression data using real-time quantitative PCR and the $2^{-\Delta \Delta C_{\mathrm{T}}}$ method. Methods 25: 402-408, 2001.

33. Gibney ER and Nolan CM: Epigenetics and gene expression. Heredity 105: 4-13, 2010.

34. Kulis $M$ and Esteller M: DNA methylation and cancer. Adv Genet 70: 27-56, 2010.

35. Nile CJ, Read RC, Akil M, Duff GW and Wilson AG: Methylation status of a single $\mathrm{CpG}$ site in the IL6 promoter is related to IL6 messenger RNA levels and rheumatoid arthritis. Arthritis Rheum 58: 2686-2693, 2008.

36. Prokhortchouk E and Hendrich B: Methyl-CpG binding proteins and cancer: Are MeCpGs more important than MBDs? Oncogene 21: 5394-5399, 2002

37. Jones PL, Veenstra GJ, Wade PA, Vermaak D, Kass SU, Landsberger N, Strouboulis J and Wolffe AP: Methylated DNA and $\mathrm{MeCP} 2$ recruit histone deacetylase to repress transcription. Nat Genet 19: 187-191, 1998.

38. Ng HH, Zhang Y, Hendrich B, Johnson CA, Turner BM, Erdjument-Bromage H, Tempst P, Reinberg D and Bird A: MBD2 is a transcriptional repressor belonging to the MeCP1 histone deacetylase complex. Nat Genet 23: 58-61, 1999.

39. Clark SJ, Harrison J and Molloy PL: Spl binding is inhibited by ${ }^{\mathrm{m}} \mathrm{Cp}{ }^{\mathrm{m}} \mathrm{CpG}$ methylation. Gene 195: 67-71, 1997.

40. Kim TW, Lee SJ, Oh BM, Lee H, Uhm TG, Min JK, Park YJ, Yoon SR, Kim BY, Kim JW, et al: Epigenetic modification of TLR4 promotes activation of $\mathrm{NF}-\kappa \mathrm{B}$ by regulating methyl-CpG-binding domain protein 2 and $\mathrm{Sp} 1$ in gastric cancer. Oncotarget 7: 4195-4209, 2016.

41. Murayama A, Sakura K, Nakama M, Yasuzawa-Tanaka K, Fujita E, Tateishi Y, Wang Y, Ushijima T, Baba T, Shibuya K, et al: A specific $\mathrm{CpG}$ site demethylation in the human interleukin 2 gene promoter is an epigenetic memory. EMBO J 25: 1081-1092, 2006.

42. Perera EM, Bao Y, Kos L and Berkovitz G: Structural and functional characterization of the mouse tescalcin promoter. Gene 464: 50-62, 2010

43. Stein L, Rothschild J, Luce J, Cowell JK, Thomas G, Bogdanova TI, Tronko MD and Hawthorn L: Copy number and gene expression alterations in radiation-induced papillary thyroid carcinoma from chernobyl pediatric patients. Thyroid 20: 475-487, 2010.

44. Man CH, Lam SS, Sun MK, Chow HC, Gill H, Kwong YL and Leung AY: A novel tescalcin-sodium/hydrogen exchange axis underlying sorafenib resistance in FLT3-ITD ${ }^{+}$AML. Blood 123: 2530-2539, 2014

45. Qureshi SA, Bashir MU and Yaqinuddin A: Utility of DNA methylation markers for diagnosing cancer. Int J Surg 8: 194-198, 2010.

46. Kim TW, Kim B, Kim JH, Kang S, Park SB, Jeong G, Kang HS and Kim SJ: Nuclear-encoded mitochondrial MTO1 and MRPL41 are regulated in an opposite epigenetic mode based on estrogen receptor status in breast cancer. BMC Cancer 13: 502 , 2013.

47. Chen B, Zeng C, Ye Y, Wu D, Mu Z, Liu J, Xie Y and Wu H: Promoter methylation of TCF21 may repress autophagy in the progression of lung cancer. J Cell Commun Signal 12: 423-432, 2018.

48. Wang Y, He T, Herman JG, Linghu E, Yang Y, Fuks F, Zhou F, Song L and Guo M: Methylation of ZNF331 is an independent prognostic marker of colorectal cancer and promotes colorectal cancer growth. Clin Epigenetics 9: 115, 2017.

49. Solomon O, Yousefi P, Huen K, Gunier RB, Escudero-Fung M, Barcellos LF, Eskenazi B and Holland N: Prenatal phthalate exposure and altered patterns of DNA methylation in cord blood. Environ Mol Mutagen 58: 398-410, 2017.

50. Han KM, Won E, Kang J, Choi S, Kim A, Lee MS, Tae WS and Ham BJ: TESC gene-regulating genetic variant (rs7294919) affects hippocampal subfield volumes and parahippocampal cingulum white matter integrity in major depressive disorder. J Psychiatr Res 93: 20-29, 2017.

51. Delcuve GP, Khan DH and Davie JR: Roles of histone deacetylases in epigenetic regulation: emerging paradigms from studies with inhibitors. Clin Epigenetics 4: 5, 2012.

52. Sarkar S, Abujamra AL, Loew JE, Forman LW, Perrine SP and Faller DV: Histone deacetylase inhibitors reverse CpG methylation by regulating DNMT1 through ERK signaling. Anticancer Res 31: 2723-2732, 2011

53. Campanero MR, Armstrong MI and Flemington EK: CpG methylation as a mechanism for the regulation of E2F activity. Proc Natl Acad Sci USA 97: 6481-6486, 2000. 
54. Tian HP, Lun SM, Huang HJ, He R, Kong PZ, Wang QS, Li XQ and Feng YM: DNA methylation affects the SP1-regulated transcription of FOXF2 in breast cancer cells. J Biol Chem 290: 19173-19183, 2015

55. Garrity PA, Chen D, Rothenberg EV and Wold BJ: Interleukin-2 transcription is regulated in vivo at the level of coordinated binding of both constitutive and regulated factors. Mol Cell Biol 14: 2159-2169, 1994.

56. Iguchi-Ariga SM and Schaffner $\mathrm{W}$ : $\mathrm{CpG}$ methylation of the cAMP-responsive enhancer/promoter sequence TGACGTCA abolishes specific factor binding as well as transcriptional activation. Genes Dev 3: 612-619, 1989.

57. Nair SS, Coolen MW, Stirzaker C, Song JZ, Statham AL, Strbenac D, Robinson MD and Clark SJ: Comparison of methyl-DNA immunoprecipitation (MeDIP) and methyl-CpG binding domain (MBD) protein capture for genome-wide DNA methylation analysis reveal $\mathrm{CpG}$ sequence coverage bias. Epigenetics 6: 34-44, 2011.

58. Fujita N, Takebayashi S, Okumura K, Kudo S, Chiba T, Saya H and Nakao M: Methylation-mediated transcriptional silencing in euchromatin by methyl-CpG binding protein MBD1 isoforms Mol Cell Biol 19: 6415-6426, 1999.
59. Patra SK, Patra A, Zhao H, Carroll $P$ and Dahiya R: Methyl-CpG-DNA binding proteins in human prostate cancer: expression of CXXC sequence containing MBD1 and repression of MBD2 and MeCP2. Biochem Biophys Res Commun 302: 759-766, 2003

60. Bird AP and Wolffe AP: Methylation-induced repression-belts, braces, and chromatin. Cell 99: 451-454, 1999.

61. Dhasarathy A and Wade PA: The MBD protein family - reading an epigenetic mark? Mutat Res 647: 39-43, 2008.

62. Ng HH, Xu RM, Zhang Y and Struhl K: Ubiquitination of histone H2B by Rad6 is required for efficient Dot1-mediated methylation of histone H3 lysine 79. J Biol Chem 277: 34655-34657, 2002.

63. Fernandez-Capetillo O, Allis CD and Nussenzweig A: Phosphorylation of histone H2B at DNA double-strand breaks. J Exp Med 199: 1671-1677, 2004.

64. Tamagawa H, Oshima T, Shiozawa M, Morinaga S, Nakamura Y, Yoshihara M, Sakuma Y, Kameda Y, Akaike M, Masuda M, et al: The global histone modification pattern correlates with overall survival in metachronous liver metastasis of colorectal cancer. Oncol Rep 27: 637-642, 2012. 